\title{
Giotto confirms reports of dumbbell-shaped nucleus
}

\section{Darmstadt}

THE notice DO NOT PANIC hung in one of the experimental areas here last week and, throughout a somewhat vertiginous approach by Giotto, the European Space Agency (ESA)'s spacecraft, to the core of comet Halley in the first few minutes of 14 March (GMT), and despite a loss of signal at closest approach, nobody did. And there, at last, was the nucleus. "It looks like a peanut", said one. "A potato", said another. And it is black.

It was the perfect day for an encounter. The solar wind was calm, with a speed of about $380 \mathrm{~km} \mathrm{~s}^{-1}$ with a steady interplanetary magnetic field of about 8 nanotesla. Earlier, there had been fears that solar activity might produce choppy electromagnetic conditions, in which case information from satellites such as the Solar Maximum Mission might have been essential to distinguish the effects of a gusty plasma environment from truly cometary phenomena.

Before the Giotto encounter, expectaions were conditioned by the two Vega encounters the previous week (see Nature 320, 97; 1986). The Soviet chief scientist, Roald Sagdeev, had brought the news that Vega 2 had shown that the nucleus looked rather like a potato of maximum length 11 $\mathrm{km}$. But there was some fear that the Vega detectors had been deceived by the variable brightness of the dust. "I'm convinced Vega saw the nucleus", said Fred Whipple, architect of the prevailing "dirty snowball" model; "there were earlier reports of a double nucleus, but the imaging people now say it's more like an irregular potato with two bright ends."

With its tenfold increase in resolution, Giotto could be expected to resolve the debate. Meanwhile, the dust specialists were perturbed but philosophical about reports from Japan of large and potentially disastrous dust particles detected by the Suisei spacecraft at the surprisingly large distance of more than $150,000 \mathrm{~km}$ from the nucleus (see Nature 320,99; 1986).

In the event, Giotto first detected effects of the comet on its environment at a distance of 7.8 million $\mathrm{km}$, with cometary ions and plasma waves becoming more and more noticeable. At this point, Giotto had entered the outer coma, a vast invisible cloud whose expansion is virtually unhindered by Halley's tiny gravity. Cometary hydrogen and oxygen atoms plus hydroxyl radicals began to be detected, the results of photodissociation of water vapour sublimed from the nucleus.

As the solar wind picks up such atoms, it is decelerated to a point (roughly corres- ponding to a 25 per cent increase in mass loading) where a bow-shock should form, with a sudden change in flow conditions. There had been some debate whether such a shock had been detected at comet Giaccobini-Zinner by the International Cometary Explorer. Pretty much on schedule, howe $\mathrm{er}$, according to principal investigator Alan Johnstone, Giotto entered a weak bow-shock (or bow-wave) at 1.15 million $\mathrm{km}$, extending along its trajectory over 0.5 million $\mathrm{km}$ or so. The spacecraft detected a shift in solar wind direction of about $15^{\circ}$, a slowing of helium ion speeds to $260 \mathrm{~km} \mathrm{~s}^{-1}$ and fluctuations in the magnetic field of up to 20 nanotesla.

The excitement became more noticeable with the first images from a distance

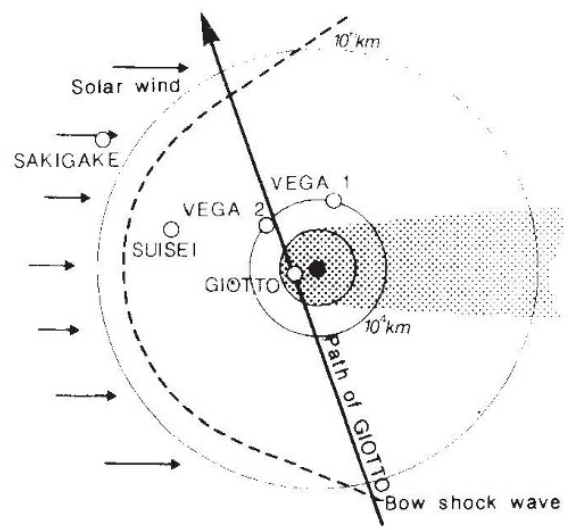

Closest approach:

$\begin{array}{lr}\text { Sakigake } & 7,000,000 \mathrm{~km} \\ \text { Suisei } & 200,000 \mathrm{~km} \\ \text { Vega 1 } & 10,000 \mathrm{~km} \\ \text { Vega 2 } & 10,000 \mathrm{~km} \\ \text { Giotto } & 500 \mathrm{~km}\end{array}$

of $750,000 \mathrm{~km}$ though, with resolution of $50 \mathrm{~km}$ or so, the nucleus was not yet visible. The atmosphere was distinctly polyglot as collaborators from many parts of the globe began to pile into the surprisingly restricted space taken up by each of the ten experimental groups. By means of a direct link to Moscow, about 30 people there were also following the encounter.

At about 0.5 million $\mathrm{km}$, the presence of cometary ions was much more pronounced. Here also, cometary chemistry began to be noticeable and, as its pick-up of heavy ions increased, the speed of the solar wind decreased further. "It's terrific, it's terrific", exclaimed one experimenter. But what about the dust? So far there was none, and principal investigator Tony McDonnell was discreetly beginning to wonder whether his impact detectors had failed. To his relief, the first particle finally arrived, detected on Giotto's front shield at about $200,000 \mathrm{~km}$.
At about $48,000 \mathrm{~km}$, the nucleus seemed to be resolved as an unchanging feature in the camera frame. A few minutes later came the first hint of trouble as the attitude sensor indicated a slight shift in the spin axis. But by now the instruments were working overtime as Giotto approached the nucleus from behind its left shoulder, as it were, and heading towards the Sun-side, from which most of the outflow of gas and dust occurs.

As Giotto's camera followed the changing relative position, its images were relayed live, every few seconds, to the watchers on the ground. A few minutes before the closest encounter, the region of the nucleus began rapidly to dominate the screen, and mottled features became apparent. It was a frankly emotional moment; the quiet voice of the scientific commentary began to shake with excitement and the assembly broke into applause. At about $5,000 \mathrm{~km}$, the magnetometers saw the magnetic field vanish - Giotto had penetrated the ionopause where electrical currents carried by electrons and ions cancel the ambient field

Almost to the second of closest encounter, at a distance of $605 \mathrm{~km}$, the images ceased and the telemetry link at the 64-m Parkes radiotelescope in Australia lost contact with the spacecraft. Tony McDonnell later said, "we seemed to hit a wall of dust a few minutes before the encounter". The impact rate had then gradually increased from the already high rate of 120 per second to the point where the spacecraft suddenly went out of alignment. But in the belief that the spinning spacecraft had been knocked into a wobbling precession, damping manoeuvres were carried out and contact re-established after $30 \mathrm{~min}$.

And the nucleus? According to the camera principal investigator Horst Uwe Keller, first indications are that it is $15 \mathrm{~km}$ long and at least $4 \mathrm{~km}$ wide, perhaps shaped like an indented potato or peanut shell. The loss of contact meant that only one side of the nucleus was seen, so that an accurate evaluation of the volume will not be possible, nor will the variation of the reflecting properties of nuclear material with Sun angle be fully known.

Nevertheless, surface features have been seen with a best resolution of $70 \mathrm{~m}$. The nucleus is "blacker than coal", according to Keller. "similar to the lowest albedos yet seen in the Solar System". He says that the surface must be at least warm enough to melt ice. Fred Whipple believes that such an appearance could arise from ice embedded with a great deal of dust including carbon or organic material.

Perhaps the major surprise of the encounter, according to chief scientist R. Reinhard, was the great inhomogeneity of dust emission. To a much greater extent than expected, the dust outflow is concentrated in jets and there are indications of nozzle-like features on the surface. A 
most important task will now be to match in situ observations of jets with those provided by the International Halley Watch network.

Analysis of mass spectrometry also proved exciting. "When we moved into the coma, we were overwhelmed by the richness of the neutral and ion spectra", said principal investigator D. Krankows$\mathrm{ki}$. The major ions turned out to be those associated with water vapour, $\mathrm{H}_{3} \mathrm{O}^{+}$and $\mathrm{H}_{2} \mathrm{O}^{+}$, with a sharp increase in density to a plateau of about $10^{3}$ per cubic centimetre at about $30,000 \mathrm{~km}$; among the neutrals, $\mathrm{H}_{2} \mathrm{O}$ reached $10^{8} \mathrm{~cm}^{-3}$ at about $3,000 \mathrm{~km}$. The total water outflow was estimated to be about $2 \times 10^{30}$ molecules per second. Compounds of carbon, nitrogen and oxygen were also seen by optical spectrometry. $\mathrm{C}_{2}$ was found to be more abundant than $\mathrm{CN}$, the latter and $\mathrm{CO}^{+}$increasing from a distance of $150,000 \mathrm{~km}$ with a sharp rise in $\mathrm{CO}^{+}$just before approach. Metal ions were also seen, though as $\mathrm{H}$. Balsinger indicated, if the nucleus is a potato it is not a salty one; little sodium was seen.

The blackness of the nucleus may be linked to another surprise reported by Tony McDonnell - the presence of an unexpected population of dust with masses as small as $10^{-17}$ grams. As Vega dust scientist J.S. Simpson remarked, most models predict a fall-off in dust masses below $10^{-12}$ grams, but Vega and Giotto have indicated substantial numbers of smaller particles. It had been thought that such particles would have been expelled from the Solar System by radiation pressure before they could be incorporated into comets. The efficiency of such processes may now have to be re-evaluated in view of the photo-properties of the nucleus discovered by Giotto.

The density of each dust particle seems to have been less than that of water, indicating a porous structure, possibly with a solid core covered with an icy mantle.

Comparing the Giotto and Vega missions, Sagdeev said that the gas, dust and particle measurements showed very good correspondence while highlighting the variability of the neighbourhood of Halley's nucleus. The Giotto measurements of in situ chemistry should be matched with Vega's remote sensing spectrometers, whose resolution might permit investigation even closer to the nucleus than Giotto.

"The science was really great", he said. "After many sleepless nights, we have to part with the comet. We feel real nostalgia now which will last beyond Halley's next apparition."

Meanwhile, Giotto is still working, although with possible damage to the camera and altitude control. It is hoped to adjust its orbit, enabling it to use the Earth as a gravitational sling in 1990 , perhaps to encounter comet Grigg-Skjellerup in 1992.

\section{Book conservation}

\section{Red faces over US pilot plant}

\section{Washington}

An explosion and fire have raised design and safety questions about a book conservation process developed by the Library of Congress (LC) that supposedly presented "absolutely no safety risks to personnel or books". The process was designed to make book paper less acidic.

The explosion occurred at a pilot facility operated by Northrop Services Inc., under contract to the National Aeronautics and Space Administration (NASA) at the Goddard Space Flight Center in Greenbelt, Maryland. The facility was designed to support start-up of a $\$ 11.5$ million Mass Deacidification Facility for LC to be constructed at Fort Detrick, Maryland.

What started as a small leak of the chemical used in the deacidication process in early December ultimately resulted in the "controlled disassembly" of the entire system by a demolition team on 21 February.

Book deterioration caused by the high acid content of paper made from wood pulp is a serious problem for all libraries. To decrease the acid content, LC developed and patented a vapour phase process using diethyl zinc (DEZ). The process works by placing books in a vacuum chamber at 20 torr, removing most of the moisture from the books, then injecting gaseous DEZ into the chamber. The DEZ reacts with sulphuric acid in the paper, forming zinc sulphate and water, and the water then reacts with additional DEZ to form zinc oxide in the paper fibres. This provides an alkaline reserve against further acid degradation. Zinc carbonate, produced by adding carbon dioxide to the reaction, also buffers the paper, as well as providing some measure of protection against photodegradation of cellulose.

While the DEZ process has advantages over other liquid processes that have been developed, DEZ is a difficult material to work with. It is pyrophoric in its liquid state at room temperature, burning on contact with air and explosively decomposing on contact with water.

On 5 December, with the book chamber in the pilot facility empty, the system was put through its operational cycle. At the point where all DEZ should have either been recovered or vented, the door to the chamber was allowed to open. The technician on duty reports seeing approximately one litre of liquid DEZ spill out of the chamber onto the ground. Automatic sprinklers extinguished the resulting fire in 8 seconds, and only minor damage resulted, but project engineers immediately shut-down the system while they attempted to discover why liquid DEZ had formed in what was supposed to be a vapour phase of the process. The best guess, according to James Robinson, acting associate director for institutional management at Goddard and chairman of the board of inquiry into the accident, is that some pipes in the gas delivery system had cooled to the point where DEZ would condense, even at low pressure.

When NASA and Northrop engineers returned to the facility early last month, they discovered a positive pressure in the recovery leg of the system. Assuming that additional DEZ or ethane, a reaction byproduct, still remained in some of the process piping, they attempted to vent the remaining gas. During this process, a "significant" explosion occurred, destroying valves, pipes and instrumentation, and compromising the ability to "safe" the system with nitrogen gas.

NASA and Northrop engineers were unable to tell whether any DEZ remained in the process piping, and, if it did, how to remove it safely. Robinson says project engineers considered punching a hole in the remaining pipes from a distance with a rifle bullet, but rejected single-point venting because all remaining DEZ might not escape. NASA and Northrop ultimately concluded that the only way to make the facility safe was to destroy it. An army demolition team set off ten shaped charges on 21 February, "disassembling" the system. The DEZ released at this point burned off in 30 seconds, but this fire spread to the wooden structure housing the system, which burned for nearly an hour. Damage was estimated at $\$ 30,000$.

Both NASA and LC are satisfied that adequate safety procedures were followed in the design of the test facility. If anything, NASA and LC officials say the nature of DEZ prompted excessive safety concerns about its handling.

But safety issues have been raised in the past about how NASA and Northrop conducted the deacidification testing. In 1982, a Northrop safety engineer was fired for insubordination after bypassing his own superiors and reporting safety concerns about the project directly to NASA.

While Robinson would not rule out the possibility that safety issues may have been neglected, he contends "the safety review was adequate... but the fact is that we have had two accidents in three months".

LC has delayed opening of bids for construction of the Mass Deacidification Facility for at least a month until the board of inquiry makes its report.

Peter Sparks, director of preservation for LC, says the pilot system was designed to point out design problems. "We learned a lot from this, but it wasn't quite the way we wanted to learn it."

Joseph Palca 